Kay P. Hradilak

Führen von IT-ServiceUnternehmen 


\section{Edition CIO}

Herausgegeben von Andreas Schmitz und Horst Ellermann

Der Schlüssel zum wirtschaftlichen Erfolg von Unternehmen liegt heute mehr denn je im sinnvollen Einsatz von Informationstechnologie. Nicht ob, sondern WIE die Informationstechnik der Motor für wirtschaftlichen Erfolg sein wird, ist das Thema der Buchreihe. Dabei geht es nicht nur um Strategien für den IT-Bereich, sondern auch deren Umsetzung - um Architekturen, Projekte, Controlling, Prozesse, Aufwand und Ertrag.

Die Reihe wendet sich an alle Entscheider in Sachen Informationsverarbeitung, IT-Manager, Chief Information Officer - kurz: an alle IT-Verantwortlichen bis hinauf in die Chefetagen.

Konsequente Ausrichtung an der Zielgruppe, hohe Qualität und dadurch ein großer Nutzen kennzeichnen die Buchreihe. Sie wird herausgegeben von der Redaktion der IT-Wirtschaftszeitschrift CIO, die in Deutschland seit Oktober 2001 am Markt ist und in den USA bereits seit 19 Jahren erscheint.

\section{Netzarchitektur - Entscheidungshilfe für Ihre Investition}

Von Thomas Spitz

\section{Management von IT-Architekturen}

Von Gernot Dern

\section{Kommunikationssysteme mit Strategie}

Von Peter Fidrich

Chefsache Open Source

Von Theo Saleck

\section{Chefsache IT-Kosten}

Von Theo Saleck

\section{Outsourcing realisieren}

Von Marcus Hodel, Alexander Berger und Peter Risi

\section{Optimiertes IT-Management mit ITIL}

Von Frank Victor und Holger Günther

Von der Unternehmensarchitektur zur IT-Governance

Von Klaus D. Niemann

IT-Controlling realisieren

Von Andreas Gadatsch

Führen von IT-Service-Unternehmen

Von Kay P. Hradilak

Weitere Titel sind in Vorbereitung. 
Kay P. Hradilak

\section{Führen \\ von IT-Service- Unternehmen}

\section{Zukunft erfolgreich gestalten}

Mit 21 Abbildungen

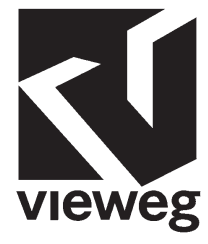


Bibliografische Information Der Deutschen Nationalbibliothek

Die Deutsche Nationalbibliothek verzeichnet diese Publikation in der Deutschen Nationalbibliografie; detaillierte bibliografische Daten sind im Internet über <http://dnb.d-nb.de> abrufbar.

Die Wiedergabe von Gebrauchsnamen, Handelsnamen, Warenbezeichnungen usw. in diesem Werk berechtigt auch ohne besondere Kennzeichnung nicht zu der Annahme, dass solche Namen im Sinne von Warenzeichen- und Markenschutz-Gesetzgebung als frei zu betrachten wären und daher von jedermann benutzt werden dürfen.

Höchste inhaltliche und technische Qualität unserer Produkte ist unser Ziel. Bei der Produktion und Auslieferung unserer Bücher wollen wir die Umwelt schonen: Dieses Buch ist auf säurefreiem und chlorfrei gebleichtem Papier gedruckt. Die Einschweißfolie besteht aus Polyäthylen und damit aus organischen Grundstoffen, die weder bei der Herstellung noch bei der Verbrennung Schadstoffe freisetzen.

1. Auflage Januar 2007

Alle Rechte vorbehalten

(C) Friedr. Vieweg \& Sohn Verlag | GWV Fachverlage GmbH, Wiesbaden 2007

Lektorat: Günter Schulz / Andrea Broßler

Der Vieweg Verlag ist ein Unternehmen von Springer Science+Business Media. www.vieweg.de

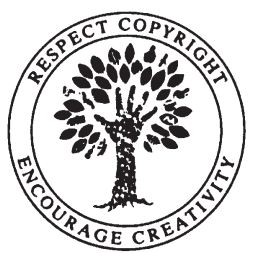

Das Werk einschließlich aller seiner Teile ist urheberrechtlich geschützt. Jede Verwertung außerhalb der engen Grenzen des Urheberrechtsgesetzes ist ohne Zustimmung des Verlags unzulässig und strafbar. Das gilt insbesondere für Vervielfältigungen, Übersetzungen, Mikroverfilmungen und die Einspeicherung und Verarbeitung in elektronischen Systemen.

Umschlaggestaltung: Ulrike Weigel, www.CorporateDesignGroup.de

Umschlagbild: Nina Faber de.sign, Wiesbaden

Druck- und buchbinderische Verarbeitung: MercedesDruck, Berlin

Printed in Germany

ISBN 978-3-8348-0260-6 


\section{Geleitwort}

Seit das Gebiet nicht mehr „Elektronische Datenverarbeitung“ genannt wird, sondern auf den Namen „Information Technology“ hört, hat es an Attraktivität deutlich gewonnen. Auch der Übergang der Funktionsbezeichnung vom „Leiter DV“ zum „IT-Manager“ war dem Ansehen sehr zuträglich. Wenn dann noch die Perspektive des „Chief Information Officers“ eröffnet wurde und die Aus- oder Neugründung von unabhängigen Servicegesellschaften anstand, die natürlich auch im Kleinstformat als Aktiengesellschaft mit Vorstand möglich wurde, konnte sich die Aufgabe mit allem vergleichen lassen, was der Markt sonst so an beruflichen Möglichkeiten bietet.

Der Internet-Hype hat mit den allseits bekannten Auswüchsen sein Übriges dazu gegeben. Kurz gesagt: IT-Services sind nicht nur modern, fortgeschrittene Technologie und fachlich attraktiv, sie bieten auch beste finanzielle Möglichkeiten und stellen ein leicht beherrschbares Managementareal dar. Wer an dieser Vorstellung festhalten möchte, sollte das Buch wieder beiseite legen - es wird keine Freude bereiten.

Wer dagegen schon länger den Eindruck hat, dass der Bereich Informationstechnologie und die damit verbundenen Dienstleistungen unwiderruflich aus den Flegeljahren herausgewachsen sind, dass sich jetzt Management an normal gewordenen Rahmenbedingungen messen lassen muss, die in anderen Industrien schon seit Jahrzehnten gang und gäbe geworden sind, der wird von der Lektüre profitieren.

Ausgangspunkt der Überlegungen zur Zukunft der IT-Services ist das Wirksamwerden normaler industrieller Rahmenbedingungen. Solche Anpassungsprozesse werden häufig als Krise wahrgenommen. Vielleicht ist es müßig, lange darüber zu diskutieren, ob es sich wirklich um eine Krise handelt oder eben nur um den schrittweisen, aber unwiderruflichen Wegfall von Sonderbedingungen, die dem Frühstadium einer Branche zuzuordnen sind. Krisen haben aber andere Ursachen und erfordern meistens auch andere Maßnahmen. Die Zukunft der IT-Services, wie sie in dem vorliegenden Text diskutiert wird, basiert gerade auf der Erkenntnis, dass die bisherigen Bedingungen zugunsten der wirtschaftlichen Normalität weggefallen sind.

Explizit bezieht sich die Veröffentlichung auf die Führung von IT-ServiceUnternehmen. Wer die Analyse und die Handlungsempfehlungen mit einem anderen Blickwinkel liest, wird schnell feststellen, dass sie ohne weiteres auch auf die Führung von IT-Bereichen in einem anderen organisatorischen Umfeld bezogen werden können. Das Credo, es sei nicht die Größe, die über den Erfolg entscheide, sondern Innovation, Professionalität und Serviceerfahrung, gilt auch für IT-Services, die nicht unabhängig am Markt angeboten werden.

Innovation ist eines der Stichworte, die auf das Leistungsspektrum der IT-Services angewandt werden. „Auf Innovation beruht die Daseinsberechtigung eines IT-Service-Unternehmens, wie es in diesem Buch beschrieben wird." (siehe Seite 115) Innovation bezieht sich aber nicht nur auf die Dienste, sondern auf die Organisation als Ganzes. In gewisser Hinsicht lässt sich 
der gesamte Text als Aufforderung zur Innovation des Unternehmens verstehen. Welche Überzeugungen, die sich in der Vergangenheit durchaus bewährt haben, führen unter den neuen Bedingungen nicht mehr weiter? Welchen Erfahrungen ist die praktische Grundlage entzogen worden? Welche neuen Orientierungspunkte sollen im IT-Management berücksichtigt werden?

Hier sollen nicht die inhaltlichen Thesen des Textes vorweg genommen werden. Aber soviel sei vorweg gesagt: wer für ein selbständiges Unternehmen der IT-Service-Industrie oder für einen mehr oder minder verselbständigten IT-Bereich die Ausrichtung der Führung überprüfen möchte, kann dies entlang der elf Dimensionen sehr gut tun, in denen der Weg in die Zukunft skizziert wird. Auch wer dem Autor nicht in allen Punkten folgen möchte, wird den detailliert und mit Sinn für die praktischen Fragen der Unternehmensführung formulierten Reifetest mit Bezug auf die Personalführung, die Entwicklung eines Verständnisses von Serviceprodukten oder das Zusammenspiel zwischen Vertrieb und Technik mit Gewinn lesen.

Köln, den 25. November 2006

Andreas Resch 


\section{Vorwort}

Der Geschäftsalltag von IT-Dienstleistern konfrontiert dessen Führungskräfte nahezu jeden Tag mit Herausforderungen, bei denen die gesammelten Erfahrungen und die bisherigen Erfolge nicht weiterhelfen. Mehr noch. Der Erfolg der Neunziger Jahre des letzten Jahrhunderts und die mit ihm gewachsenen Überzeugungen können jetzt lebensgefährlich für IT-ServiceUnternehmen werden.

Service- und Vertriebsmanager in der IT-Dienstleistungsbranche müssen heute in einem Markt bestehen, der scheinbar nur noch Preisdruck und wachsende Austauschbarkeit kennt. Ganze Marktsegmente stagnieren oder schrumpfen. Die Großunternehmen der Branche suchen ihr Heil, indem sie Wett-bewerber kaufen und größer und größer werden wollen, ohne dass dieses aber den meisten von ihnen zu einer guten Rendite verhilft. Aus Asien nahen IT-Dienstleister mit gewaltigen Ressourcen und scheinbar unschlagbaren Preisvorteilen.

Wie kann ein IT-Service-Unternehmen trotz dieser Rahmenbedingungen ertragreich gedeihen und wachsen? Denn es gibt auch heute ertragsstarke IT-Service-Unternehmen. Es gibt margenträchtige Projekte und prosperierende Kundenbeziehungen, in denen die Kunden hochzufrieden sind und die Dienstleister sehr gute Margen erzielen.

Hinter diesen ermutigenden Erfahrungen stecken gemeinsame Muster, die in diesem Buch als Service Design und als Engineeringstärke beschrieben werden. Zum einem reichen heute wettbewerbsfähige Kosten und solide Prozessbeherrschung allein nicht mehr aus, um im IT-Service-Markt hervorzutreten. Es wird heute unerlässlich, IT-Serviceprozesse hinsichtlich des größten Kundennutzens zu designen und zu führen. IT-Services müssen zu einer positiv unvergesslichen Kundenerfahrung werden. Zum anderen müssen IT-Service-Unternehmen realisieren, dass in Zukunft nicht einfach die größten Unternehmen den größten Erfolg haben werden, sondern die Unternehmen, die über die meisten Topkräfte sowohl hinsichtlich EngineeringVertrieb, Projektmanagement als auch Technologie-Können verfügen.

Wenn Vertriebs- und Servicemanager die Zukunftschancen ergreifen wollen, die in Service Design und Engineeringstärke liegen, dann müssen sie sich zuerst ihrer eigenen gewachsenen Grundüberzeugungen bewusst werden. Und sie müssen sich die Entwicklung ihrer Branche verdeutlichen, die unumkehrbar ihre „goldenen Zeiten“ hinter sich gelassen hat. Hiervon handeln die ersten beiden Kapitel des Buches. Jedem Vertriebs- und Servicemanager muss klar werden, dass er mit geliebten Überzeugungen brechen muss, damit er und sein Geschäft eine Zukunft haben.

Service Design und Engineeringstärke als Fokus bleiben Worthülsen, wenn sie nicht sämtliche Unternehmensbereiche ausdauernd prägen. Was hier konkret zu tun ist, ist Gegenstand des dritten und vierten Kapitels des Buches: Wie entwickle ich überragende Dienstleistungsprodukte, wie erschließe ich dauerhaft ertragsstarke Kundenbeziehungen, wie biete ich faszinierende 
Karrierepfade, wie organisiere ich Wachstum ohne Erstarrung und wie führe ich ein Unternehmen, das die „Besten der Besten“ an Bord hat? Jedes dieser Themen wird „von der Praxis für die Praxis“ mit einer Vielzahl von praktischen Beispielen und Checklisten beschrieben.

Der Schlüssel zur Zukunft liegt nicht in neuen „Killer“-Technologien, sondern im Führen von Teams, im Design von überragenden Serviceprodukten und in der Entwicklung von Kundenbeziehungen. Diese Erkenntnis macht auch Mut für den längerfristigen Ausblick im letzten Kapitel des Buches. Ein Verschwinden der klassischen IT-Services wird kein Ende für die Dienstleistungen bedeuten.

\section{Danksagung}

Das Schreiben dieses Buches war auch ein wunderbarer Prozess, Freundschaften zu vertiefen und interessante Menschen, vor allem natürlich aus der IT-Branche, kennenzulernen. Ivo Karzel war nicht nur einer der Ersten, der nach mir von diesem Projekt begeistert war, er begleitete auch das gesamte Buch durch all seine Versionen als kritischer Mitdenker und Mitleser. Ohne Ivo Karzels Hilfe hätte es dieses Buch in dieser Form nicht gegeben. Besonderer Dank gilt auch Herrn Damian Sicking, der als journalistischer Profi und Branchenkenner offen und kritisch dieses Buchprojekt begleitete und zum Glück nie scheute, Klartext zu reden. Herzlich zu danken habe ich auch Frau Heike Findeis, Frau Carolina Lebedies, Herrn Rolf Kleinwächter, Herrn Tim Lüdke, Herrn Andreas Mayer und Herrn Wolfgang Stübich, die mich mit wertvollen Hinweisen und spannenden Diskussionen beim Schreiben dieses Buches großartig unterstützten.

Lieben Dank an meine Frau Christine für ihre Geduld, ihren gesunden Menschenverstand, das viele Korrekturlesen und für ihre Ermutigungen. 


\section{Inhaltsverzeichnis}

Geleitwort..............................

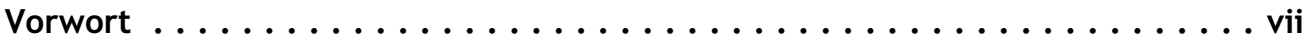

\section{Brechen Sie aus Ihren Überzeugungen aus}

„Der Fluch der Goldenen Neunziger". . . . . . . . . . . . . . . . . . . . 1

Überzeugungen, die in Stein gemeißelt sind $\ldots \ldots \ldots \ldots \ldots \ldots \ldots$

\section{IT - eine Industrie hat sich normalisiert}

2.1 IT ist eine Commodity - wird IT eine Utility $\ldots \ldots \ldots$. . . . . . . . . . 5

Kommodisierung prägt die Informationstechnologie . . . . . . . . . . . . 5

Stetiger Preisverfall ist der bestimmende Trend der Computerhardware . . . . . . . . 5

Warum der stetige Preisverfall in der „Natur der Sache“ liegt . . . . . . . . . . . . 8

Wachsender Preisdruck auch im Softwarebereich . . . . . . . . . . . 8

Die Globalisierung erhöht den Preisdruck ... . . . . . . . . . . . . . . . 9

... und ebnet den Weg für Szenarien, die die gesamte heutige Computerwelt

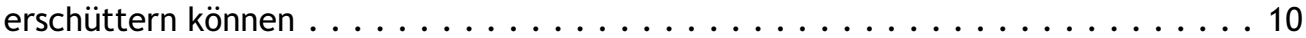

Existentielle Folgen für IT-Anbieter $\ldots \ldots \ldots \ldots \ldots \ldots \ldots$

"Computing on Demand" - die nächste Welle? . . . . . . . . . . . . . . . 13

Wie realistisch ist die Umsetzung von „Computing on Demand“? . . . . . . . . . . . 14

Stagnation in Deutschland - IT als Kostenfaktor . . . . . . . . . . . . . . 16

Fazit ............................. 17

2.2 Was sind eigentlich IT-Services? . . . . . . . . . . . . . . . . . 19

IT-Services sind vielfältig und besonders . . . . . . . . . . . . . . . . 19

Unklarheiten in der Definition . . . . . . . . . . . . . . . . . . 20

Zwei Servicewelten . . . . . . . . . . . . . . . . . . . . 21

Drei komplementäre Geschäftsfelder in den Infrastruktur-Services . . . . . . . . . . 21

Kommodisierung und „Computing on Demand“ betreffen auch die IT-Services . . . 23

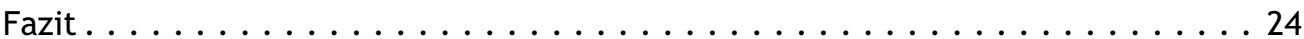




\section{Werden Sie ein Engineering-Unternehmen}

3.1 Engineeringstärke und Service Design sind entscheidender als Größe . . . . 25

IT-Services kommodisieren, aber verschwinden nicht . . . . . . . . . . . 25

Größe oder Nische? . . . . . . . . . . . . . . . . . . . . . . . . . . 25

„Größe an sich" ist selten ein Vorteil! . . . . . . . . . . . . . . . . . . . 27

Klasse statt Masse . . . . . . . . . . . . . . . . . . . 28

Mindestgrößen stellen keine ernsthaften Eintrittsbarrieren dar . . . . . . . . . . . . 28

Konzentration auf Engineeringstärke und Service Design . . . . . . . . . . . . . . 28

Den Preiswettbewerb relativieren . . . . . . . . . . . . . . . . . . . . 29

Die Regeln des Geschäftes ändern . . . . . . . . . . . . . . . . . . . . . . . 29

3.2 Forschung und Entwicklung „on the road“. . . . . . . . . . . 33

Die Chance aus dem Frust . . . . . . . . . . . . . . . . 33

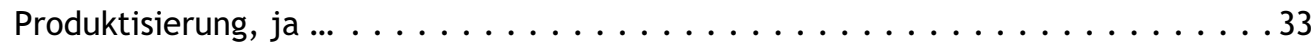

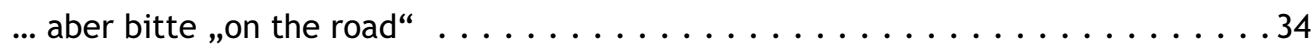

Zwei Typen von IT-Serviceprodukten . . . . . . . . . . . . . . . . . 34

IT-Engineering-Produkte . . . . . . . . . . . . . . . . . 35

Entwicklung von Consulting- und Engineering-Produkten . . . . . . . . . . 36

Betriebsprodukte . . . . . . . . . . . . . . . . . . . . 41

Die Entwicklung von Betriebsprodukten: Service Design. . . . . . . . . . . . . . 42

Organisation der Produktentwicklung . . . . . . . . . . . . . . . 45

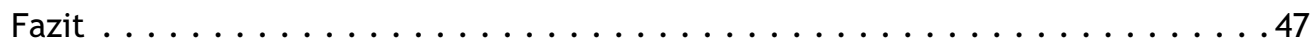

3.3 Servicevertrieb: Reißen Sie Wände ein . . . . . . . . . . . . . . . . .49

Ein klassisches Rollenspiel . . . . . . . . . . . . . . . . . . . . . . . . . . .49

Multiplizieren der Vertriebskraft: Die Serviceorganisation muss mit verkaufen. . . . . 50

Der Vertrieb paukt „Services“. . . . . . . . . . . . . . . . . . . . . 51

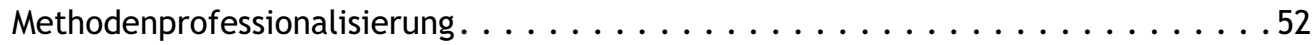

Mittelfristige Kundenentwicklung - durch Fokus besser sein . . . . . . . . . . . . 53

Vertriebstaktik - „Handwerk hat goldenen Boden“ . . . . . . . . . . . . . 60

Vertriebsmanagement mit CRM-Systemen . . . . . . . . . . . . . . . 61

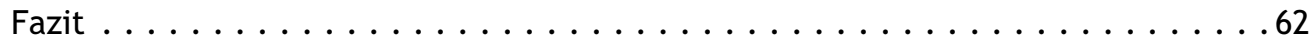


3.4 „Human Branding“ und Marketing der „Besten Praxis“. . . . . . . . . 63

Markenbildung bei IT-Service-Unternehmen . . . . . . . . . . . . . 63

Die Stunde der Wahrheit - wie werden Sie wahrgenommen? . . . . . . . . . 63

Wofür sollte das „Human Brand" eines IT-Dienstleisters stehen?. . . . . . . . . . . . 64

Markenumsetzung - Keine Kompromisse in den Details . . . . . . . . . . . . . 67

„Marketing der besten Praxis“ . . . . . . . . . . . . . . . . . . . . 67

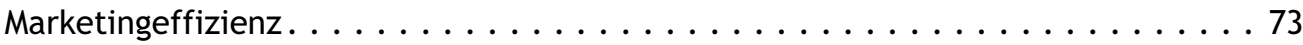

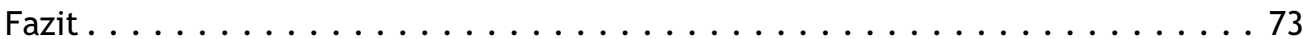

3.5 Personal: Die Besten gewinnen, fördern und entwickeln . . . . . . . 75

Menschen der IT-Service-Branche . . . . . . . . . . . . . . . . . . . . . . . 75

Wie gewinne ich Top-Engineers und Top-Verkäufer? . . . . . . . . . . . . . 76

Der „Marschallstab im Tornister“ . . . . . . . . . . . . . . . . . . . . . . . 78

Zwei Karrierepfade in den Services. . . . . . . . . . . . . . . . . . . . . . . . 79

Vertrieb: Mit der Kundenbasis wachsen . . . . . . . . . . . . . . . . 82

Was macht den Schritt von einem Bereichsleiter zu einem Geschäftsführer aus? . . . 85

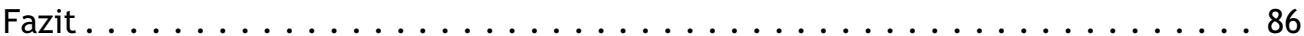

3.6 Vergütung: Bezahlen Sie für die persönliche Wertschöpfung . . . . . . 8 87

Ein paar Grundregeln . . . . . . . . . . . . . . . . . . . . . . . . . . . 87

Service-Vergütung: Weg von Stunden hin zu Euros und zu Meilensteinen . . . . . . . 88

Vertrieb: „The Sky is the limit“ . . . . . . . . . . . . . . . . . . . . 90

Vergütung der Bereichsleiter - der Bereich und das Ganze . . . . . . . . . . . . . 93

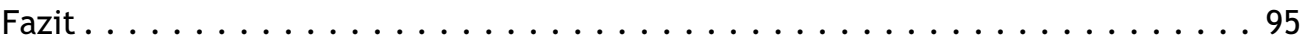

3.7 Fokussierte Beschaffung und Partnerschaftsnetze . . . . . . . . . . . . 97

Make or Buy-Entscheidungen hinsichtlich Können und Kapazität . . . . . . . . . . . . 97

Entscheidungen zum Fokus in den Technologiepartnerschaften . . . . . . . . . 98

Auswahl und Aufbau von Servicepartnerschaften . . . . . . . . . . . . . . . . . . 99

Interne Besetzung von Projekten auf Basis einer Skill- und Projektdatenbank . . . . 100

Fazit . . . . . . . . . . . . . . . . . . . . . . . . . . . 102

3.8 Organisation: Regionen, Kunden, Projekte, Produkte und Basiswissen . . 103

Zwei Fragen am Anfang . . . . . . . . . . . . . . . . . . . . . 103

Auf was richte ich die Organisation aus? . . . . . . . . . . . . . . . 103

Exkurs: Die „Fallstricke der Größe“ . . . . . . . . . . . . . . . . . . . . . 109

Wie fördert die Organisation Wachstum ohne Erstarrung? . . . . . . . . . . . . . 111

Fazit . . . . . . . . . . . . . . . . . . . . . . . . . . . . . . . . . . 114 
3.9 Innovations- und Qualitätskultur - vom Schlagwort zur Realität. . . . . . 115 Innovations- und Qualitätskultur . . . . . . . . . . . . . . . . . 115 „Ein Schuss Paranoia“ und Bescheidenheit . . . . . . . . . . . . . . . . 117

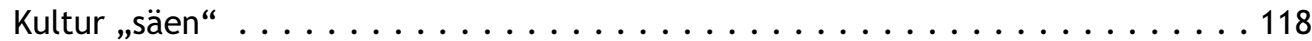
Mergers \& Acquisitions- die Königsdisziplin der Kulturarbeit . . . . . . . . . . . . 118

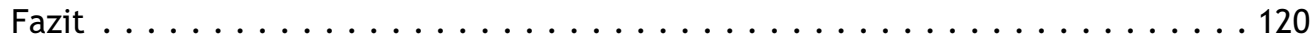

3.10 IT-Service-Controlling - von unten nach oben . . . . . . . . . . 121 Das Controlling auf die Füße stellen. . . . . . . . . . . . . . . . . . . . 121 Vorschaurechnungen und zeitnahe Ist-Abrechungen für Kunden und Teams . . . . . 122

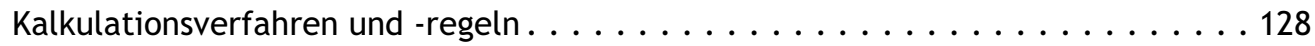
Die operative Businessplanung nach Kunden, Produkten . . . . . . . . . . . . . 129 Weitere Controllingthemen: Systematiken und spannende Analysen. . . . . . . . . . 131 Fazit . . . . . . . . . . . . . . . . . . . . . . . . . . . . . 131

3.11 Management: Weitgehende Delegation und Führen „von der Front“ . . . .133 Eine Hochleistungsorganisation führen . . . . . . . . . . . . . . . . . . 133

Führen durch weitgehendes Delegieren und durch Orchestrieren. . . . . . . . . 133 „Heroische Führung“, ... . . . . . . . . . . . . . . . . . . . . . . 135

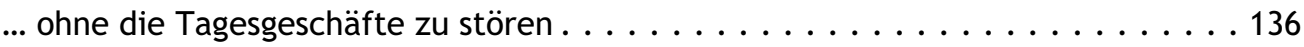

Führen mit Managementberatern? . . . . . . . . . . . . . . . . . . . . . . 137

Führen in der Krise . . . . . . . . . . . . . . . . . . . . . . . . . . . . . . . . . 139

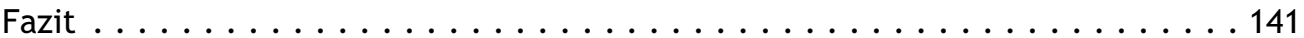

\section{Ausblick: Werden Sie Servicearchitekt und Utility Engineer}

Ausblick 2015: Das Ende der IT wie wir sie kennen? . . . . . . . . . . . . . . . . 143

Die Richtung stimmt, aber die Zukunft wird nicht monolithisch sein . . . . . . . . 144 Wie sich der Markt bis 2015 verschieben wird . . . . . . . . . . . . . . . . . 144 Die Chancen für IT-Service-Unternehmen . . . . . . . . . . . . . . . . . 146 Noch mehr Chancen: Facility Management . . . . . . . . . . . . . . . . 148 Fazit . . . . . . . . . . . . . . . . . . . . . . . . . . . . . . . . 149 


\section{Anhang}

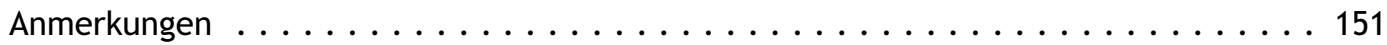

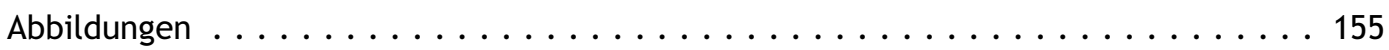

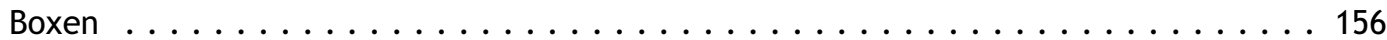

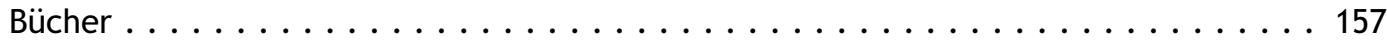

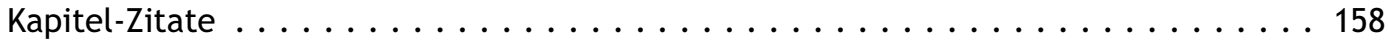

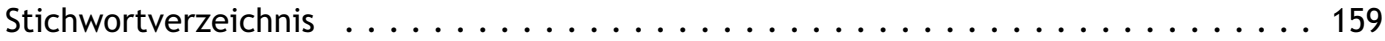

